syndrome: Analysis of 19 Caucasian patients with long-term follow-up. Epilepsia 2012 June;53(6):e102-e105). (Respond: Alberto Spalice, Child Neurology Division, Department of Pediatrics, Policlinico Umberto L Sapienza University of Rome, Viale Regina Elena 324, 00161, Roma, Italy. E-mail: childneurology.sapienzaroma@live.it).

COMMENT. More than half of children with SS have FS and/or AFS, and more than half of those with FS develop epilepsy, temporal lobe type in $40 \%$. Seizures in SS are usually responsive to AED monotherapy.

\title{
WISC-IV IN DETECTION OF EPILEPSY-RELATED COGNITIVE IMPAIRMENTS
}

Researchers at Alberta Children's Hospital Research Institute, Calgary, AB, Canada, and Epilepsy Center, New York University, NY analyzed WISC-IV scores from 212 children, 106 with epilepsy (46 girls, 60 boys; mean age 11 years), and 106 controls matched for age, gender, ethnicity, and parental education. Full Scale IQ for the epilepsy group was significantly lower than for controls; $36.8 \%$ had FSIQ $<70$ vs $<1 \%$ controls. In children with epilepsy, Working Memory and Processing Speed Index scores were lower than those for Verbal Comprehension and Perceptual Reasoning $(p<0.01)$. Scores were highest on visual and verbal subtests measuring reasoning skills such as Matrix Reasoning, and lowest on Coding. The Coding subtest identified the most children $(28.3 \%)$ with low scores, and the Similarities subtest identified the fewest (16\%). Later age at onset and shorter epilepsy duration were both correlated with higher WISC-IV FSIQ and index scores. Number of AED trials but not seizure frequency was inversely correlated with FSIQ and index scores. FSIQ, index scores, and subtest scores were not related to left or right-hemisphere seizure foci or to MRI findings. The WISC-IV is useful in detection of cognitive deficits related to childhood epilepsy. (Sherman EMS, Brooks BL, Fay-McClymont TB, MacAllister WS. Detecting epilepsy-related cognitive problems in clinically referred children with epilepsy: Is the WISC-IV a useful tool? Epilepsia 2012 June;53(6):1060-1066). (Respond: Elizabeth MS Sherman PhD, Neurosciences Program, Alberta Children's Hospital, 2888 Shaganappi Trail NW, Calgary, T3B 6A8 AB Canada. E-mail: Elizabeth.sherman@albertahealthservices.ca).

COMMENT. Cognitive impairments in children with epilepsy are secondary to the effects of seizures and AEDs and are sometimes primary, associated with abnormal brain circuitry of epilepsy and predating the onset of seizures. The WISC-IV intelligence test is sensitive to cognitive impairments in children with epilepsy but is not sufficient for measuring some cognitive problems such as memory, sustained attention, executive function, and language deficits often present in children with epilepsy (MacAllister WS, Sherman EMS. Evaluation of children and adolescents with epilepsy. In Barr W, Morrison C (Eds) Handbook of the Neuropsychology of Epilepsy. New York, Springer 2012. In press.) Evaluation of the IQ and other neuropsychological functions is an important aspect of the comprehensive management of childhood epilepsy and an essential component of an Epilepsy Center. 
Cognitive effects of interictal epileptiform EEG discharges. Simultaneous video-EEG recordings and cognitive testing in 188 children with epilepsy found that the association between frequent epileptiform activity and cognitive function, although less pronounced, is comparable to impairment of global cognitive function, central processing speed, and memory function that accompanies short nonconvulsive seizures. (Nicolai $J$ et al. Epilepsia 2012 June;53(6);1051-1059). Transient cognitive impairment (TCI) is demonstrated in about $50 \%$ of patients who show epileptiform activity during testing Whether TCI influences school performance is unclear and is not generally an indication for AED therapy. The EEG activation or suppression effect of cognitive tasks is a phenomenon to be recognized in the evaluation of TCI effects on learning. (Binnie CD. Lancet Neurol 2003;2:725-730).

\section{MOVEMENT DISORDERS}

\section{GABA-ERGIC DYSFUNCTION IN TOURETTE SYNDROME}

Researchers at the FDA, Silver Spring, MD; Department of Neurology, University of Pittsburgh Medical School, PA; National Institute of Mental Health, NIH, Bethesda, MD; and other centers used a PET study to assess the involvement of the GABA-ergic system in 11 adult Tourette syndrome patients compared to 11 healthy controls. Structural MRI scans provided an anatomical framework for the PET data analysis. Tourette patients had decreased binding of GABA receptors in the ventral striatum, globus pallidus, thalamus, amygdala and right insula. Increased binding of GABA receptors was found in the bilateral substantia nigra, left periaqueductal grey, right posterior cingulate cortex and bilateral cerebellum. These findings are consistent with the hypothesis that basal ganglia and thalamus circuits are disinhibited in Tourette syndrome. (Lerner A, Bagic A, Simmons JM, et al. Widespread abnormality of the gamma-aminobutyric acid-ergic system in Tourette syndrome. Brain 2012 June;135(6):1926-1936). (Response: Alicja Lerner, MD, PhD, FDA, 10903 New Hampshire Ave, Silver Spring, MD 20993. E-mail: alicja.lerner@hhs.gov).

COMMENT. The global functional disorganization of cortico-basal ganglia networks found in patients with TS agrees with the hypothesis of a functional immaturity of these circuits, contributing to a clinical heterogeneity of TS, including association with ADHD and OCD. Severity of OCD is correlated with functional abnormalities in associative and limbic networks, orbito-frontal and prefrontal dorsolateral cortices. (Worbe Y et al. Functional immaturity of cortico-basal ganglia networks in Gilles de la Tourette syndrome. Brain 2012 June;135(6):1937-1946).

Omega-3 Fatty Acids and TS. A double-blind, placebo-controlled, add-on trial of omega-3 FA in 33 children and adolescents with TS showed no significant reduction in frequency of tics, but significantly more subjects on omega-3 were benefited by a reduction in tic severity and related impairments. The rate of ADHD comorbidity was significantly lower in the omega-3 group than placebo. Mean end dose of omega-3 was $4074 \mathrm{mg} / \mathrm{d}$ and much higher than that generally advocated for ADHD patients (600 mg/d). (Gabbay V et al. Pediatrics 2012 June;129:e1493-e1500). 